\title{
Study of impurity behaviour for first magnetic configuration changes in W7-X plasmas by means of PHA spectra
}

\author{
Agata Czarnecka ${ }^{\mathrm{a}}$, Tomasz Fornal ${ }^{\mathrm{a}}$, Marta Gruca ${ }^{\mathrm{a}}$, Natalia Krawczyk ${ }^{\mathrm{a}}$, Monika Kubkowska ${ }^{\mathrm{a}}$ Sławomir

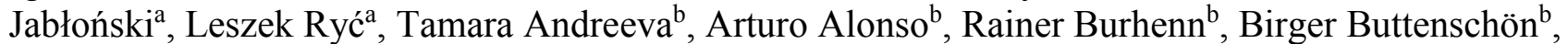 \\ Andreas Dinklage ${ }^{\mathrm{b}}$, Marcin Jakubowski ${ }^{\mathrm{b}}$, Maciej Krychowiak ${ }^{\mathrm{b}}$, Ralph König ${ }^{\mathrm{b}}$, Novimir Pablant ${ }^{\mathrm{c}}$, \\ Henning Thomsen ${ }^{\mathrm{b}}$, Daihong Zhang ${ }^{\mathrm{b}}$ and the W7-X team
}

\author{
anstitute of Plasma Physics and Laser Microfusion, Hery 23, 01-497 Warsaw, Poland \\ ${ }^{b}$ Max Planck Institute for Plasma Physics, 17491 Greifswald, Germany \\ 'Princeton Plasma Physics Laboratory, USA
}

\begin{abstract}
Transport properties of impurities in stellarators are sensitive to potential variations on flux-surfaces, and thermodynamic forces from the bulk plasma which might be affected by changes in magnetic field properties. In this contribution, the effect of planar coil current changes on the core impurity behavior in the first operating phase of Wendelstein 7-X (W7-X) is described. Hydrogen plasmas, at different heating power levels were investigated showing Core-Electron-Root-Confinement (CERC) conditions, characterized by peaked $\mathrm{T}_{\mathrm{e}}$ profiles and positive radial electric fields in the plasma core. Impurity spectra in the $\mathrm{x}$-ray emission range between 1 and $10 \mathrm{keV}$ were measured by the Pulse Height Analysis (PHA) diagnostic. The line-integrated measurements of transitions in sulphur, chlorine and argon are in the focus of this analysis. First observations indicate that the scans of the magnetic configuration did not affect strongly the impurity concentration in the core. Clear differences in the impurity content, however, have been observed when the ECRH power was changed, which affects the bulk plasma properties.
\end{abstract}

Keywords: plasma impurities, stellarator, soft X-ray plasma diagnostic, Pulse Height Analysis.

\section{Introduction}

The first operational phase (OP1.1) at the large superconducting stellarator device Wendelstein $7-\mathrm{X}$ (W7-X) allowed the integral commissioning of machine components, installed diagnostics and plasma control [15]. W7-X is a modular advanced stellarator with optimized magnetic field properties. This optimization includes the high quality of vacuum magnetic surfaces, good finite beta equilibrium and MHD-stability properties as well as a substantial reduction of the neoclassical transport in the $1 / v$ regime and bootstrap current decrease. In HELIAS devices [6] (W7-X is the first fully optimized Heli and axis advanced stellarator) the bootstrap current is kept small to avoid unfavorable changes of the edge rotational transform $[2,7]$ during the $\mathrm{L} / \mathrm{R}$ time. Plasma properties in stellarators (e.g. energy and particle confinement times), are expected to change depending on the choice of the magnetic field configuration. Possible existence of potential variations on flux-surfaces [8] and thermodynamic forces from the bulk plasma (in particular radial electric fields) will have an impact on the core impurity transport. Slight changes in the edge rotational transform (ledge) are expected to change the topology of the intrinsic magnetic islands in the scrape-off layer (SOL) [9], thus affecting impurity sources [10]. Changes in the central iota profile can alter the core impurity transport.

In the first campaign of the W7-X, a small variation of the effective helical ripple was conducted in order to study effects on the core confinement in plasmas [11]. The measured differences in helium ion decay time reflected differences in global particle confinement stemming from changes of edge magnetic island position [10]. In this contribution, the impact of W7-X magnetic configuration scans on core impurity behaviour is investigated by means of PHA spectra.

\section{Experimental setup}

Experiments were performed with a limiter configuration in hydrogen plasmas at $B_{0}=2.5 \mathrm{~T}$. Five discharges each with a different value for the iota on the magnetic axis $(\mathfrak{t})$ were analyzed. Fig. 1 shows typical time traces for the heating and plasma parameters during the 5 plasma pulses. The time traces are nearly identical for the 5 configuration scans with different iota. From top to bottom one can observe: different electron cyclotron resonance heating $(\mathrm{ECRH})$ power levels in X2 mode $\left(\mathrm{P}_{\mathrm{ECRH}}\right.$ at $2 \mathrm{MW}, 0.6 \mathrm{MW}$ and $\left.1.2 \mathrm{MW}\right)$; response of line-integrated electron densities $\left(n_{e}\right)$ from the interferometer [12]; electron temperature $\left(\mathrm{T}_{\mathrm{e}}\right)$ from ECE diagnostic [13]; and in the last graph central ion temperature $\left(\mathrm{T}_{\mathrm{i}}\right)$ derived from the $\mathrm{x}$-ray imaging crystal spectrometer (XICS) [14]. During the experiment maximum core plasma values of $\mathrm{T}_{\mathrm{e}} \sim 6 \mathrm{keV}, \mathrm{T}_{\mathrm{i}} \sim 1.7 \mathrm{keV}$ at densities $n_{e} \sim 1.6 \times 10^{19} \mathrm{~m}^{-2}$ were achieved. Pulse lengths of all analyzed discharges were $1.2 \mathrm{~s}$. Scans of magnetic configurations were performed by variations of the planar coil currents. Fig. 2 shows simulated Poincare plot of the vacuum magnetic field at the bean shaped symmetry cross section $\left(\phi_{\text {tor }}=274^{\circ}\right)$ within the vessel. The radial position of the stellarator $\mathrm{l}=5 / 6$ magnetic islands chain (with poloidal and toroidal mode numbers of $\mathrm{m}=5$ and $\mathrm{n}=6$ ), as it is presented in Fig. 2, was influenced by changing the iota on the magnetic axis in 5 
steps between $\mathfrak{t}=0.796$ and $\mathfrak{t}=0.816$. At increased iota, the $5 / 6$ islands were moved inwards. The last closed flux surface (LCFS) at the effective minor radius of $\mathrm{a}=0.49$ $\mathrm{m}$ was defined by five graphite limiters located at the inboard side of the plasma vessel $[1,2,15]$. The effective helical ripple $\varepsilon_{\text {eff }}$ also changed and varied from $0.7 \%$ to $1.4 \%$. In the configuration, for which the neoclassical ripple is significantly larger, is expected to have a lower bootstrap current.

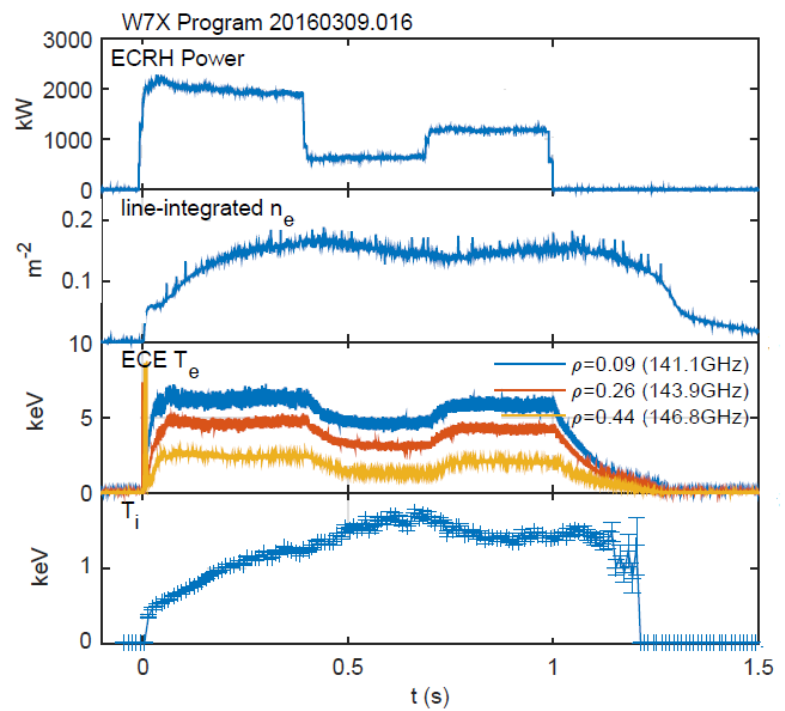

Fig. 1 A typical time evolution of the ECRH power and corresponding line integrated electron density, electron temperature for different radius/frequency correspondence, and ion temperature in the W7-X OP1.1 plasmas (shown: PID 20160309.016).

Impurity data analysis were performed using the measurements from the Pulse Height Analysis (PHA) system installed at W7-X [16-20]. The PHA diagnostic is designed to measure soft $\mathrm{X}$-ray radiation in the energy range of $0.25-20 \mathrm{keV}$. This diagnostic is dedicated for delivering information about the impurities content in the plasma, $\mathrm{T}_{\mathrm{e}}$ and estimation of $\mathrm{Z}_{\mathrm{eff}}[16,21]$. As presented in [19], the PHA diagnostic was commissioned during OP1.1 but only one energy channel of the PHA system has been optimized to deliver well-resolved spectra. Therefore, the presented results were collected by the PHA energy channel equipped with SDD detector covered by $8 \mu \mathrm{m}$ thick beryllium (Be) window and additionally $25 \mu \mathrm{m}$ of Be filter. Assuming the 1/e-decay as low energy border for the transmission window the observed energy range for channel 1 was from $900 \mathrm{eV}$ to $10 \mathrm{keV}$. The detector line-of-sight is located $\sim 8.5 \mathrm{~cm}$ below the plasma center, with a diameter of $3.5 \mathrm{~cm}$ for a pinhole size equal to $1.2 \mathrm{~mm}$. The pinhole is placed at a distance of about $7 \mathrm{~m}$ from the plasma center and the SDD detector $1 \mathrm{~m}$ behind. The PHA diagnostic line-ofsight is presented in Fig.2. The shortest time of spectrum collection was set to $100 \mathrm{~ms}$, which determines the temporal resolution of the PHA system. The choice of this value was a compromise between the quality of collected spectra (determined by statistics) and the temporal resolution of diagnostics. The energy resolution is determined by statistical fluctuations the charge generation in semiconductor detectors (determined by Fano factors) and the electronic noise. The electronic noise can be reduced by filtering input signals in the digital signal processor (DSP) of the PHA. Optimisation was performed by setting the proper value of peaking time (during OP1.1 it was $1 \mu \mathrm{s}$ ). The DSP used was very effective in removing pile-up events. The input X-ray flux is regulated by the use of adjustable slits and the counting rate is kept at the level of up to $300 \mathrm{kHz}$, what ensures reduced pile-up effect, which was at level of 10$15 \%$.
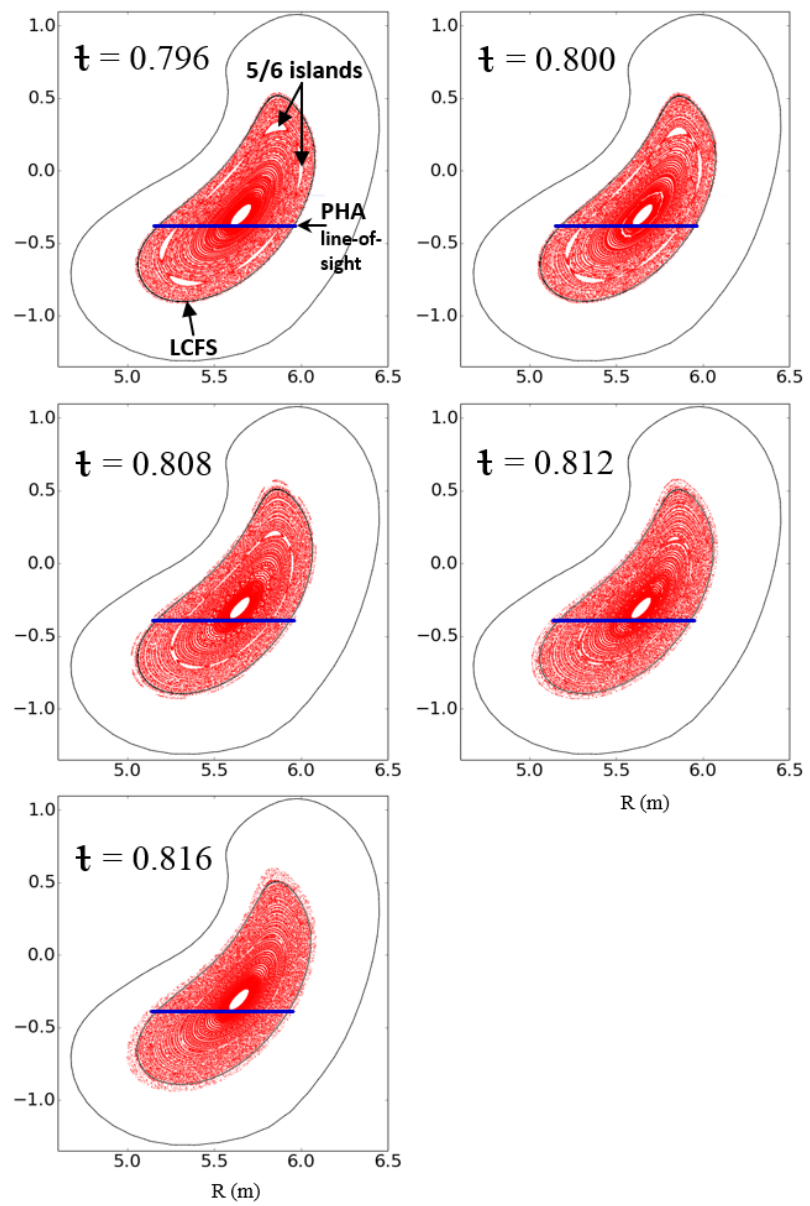

Fig. 2 Poincare plots of the vacuum magnetic field (red) at the plane of the PHA diagnostic $\left(\phi_{\text {tor }}=274^{\circ}\right)$, within the vessel (black solid line), calculated for different magnetic configurations (iota-scan). The horizontal-axis reflects major radius $(\mathrm{R})$ of the $\mathrm{W} 7-\mathrm{X}$ stellarator. The line of sight of the PHA diagnostic is indicated as the blue horizontal line.

\section{Results and discussion}

Fig. 3 shows time integrated spectra for the pulses with the scan of the OP1.1 magnetic configurations. The PHA technique allows the measurement of the intensity of both the continuum and line radiation. The He-, and H-like Sulphur (S), Chlorine (Cl) and Argon (Ar) resonance lines, with measured full width at half maximum, FWHM 140 eV at $3.1 \mathrm{keV}$, are observed in the measured spectra. All lines registered so far by the PHA diagnostic are listed in [20]. Ar is an impurity species which has been injected by the main gas inlet system into the W7-X vacuum chamber just before the plasma start (in order to derive the $\mathrm{T}_{\mathrm{i}}$ profile by means of an imaging spectrometer [22, 23]. The number of injected Ar particles from the the main gas inlet system was the same in each of the analyzed discharges. The 
source of the intrinsic impurity such as $\mathrm{S}$ and $\mathrm{Cl}$ could originate from plastic material accidentally left in the plasma vessel. The presence of $\mathrm{S}$ and $\mathrm{Cl}$ in the plasma is also confirmed by the High Efficiency XUV Overview Spectrometer system (HEXOS) [24]. Both elements were found in a surface layer analysis of the W7-X limiter [25]. Similar impurities were observed also at the tokamaks JET [26] or Alcator C [27].

The quantitative determination of impurity concentration from PHA spectra is the subject of ongoing investigation. In the following analysis we focus on the line-integrated measurements of the most intense Ar XVII at $3.140 \mathrm{keV}, \mathrm{S} \mathrm{XV}$ at $2.460 \mathrm{keV}$, and $\mathrm{Cl} \mathrm{XVI}$ at $2.789 \mathrm{keV}$ lines.



Fig. 3 Integrated PHA spectra for the discharges (PID 20160309.016, 018, 024, 026, 029) with different values of iota on the magnetic axis in W7-X plasmas plotted in the energy range $0-5 \mathrm{keV}$.

Experimentally, the intensity of a spectral peak of a selected impurity is determined by the number of counts under the peak after subtraction of the continuum contribution (consisting of both free-free and free-bound radiation). In Fig. 4 intensities of the impurity lines taken from time integrated (all over 3 ECRH-phases) PHA spectra are presented as a function of the iota on the magnetic axis $(\mathfrak{t})$.

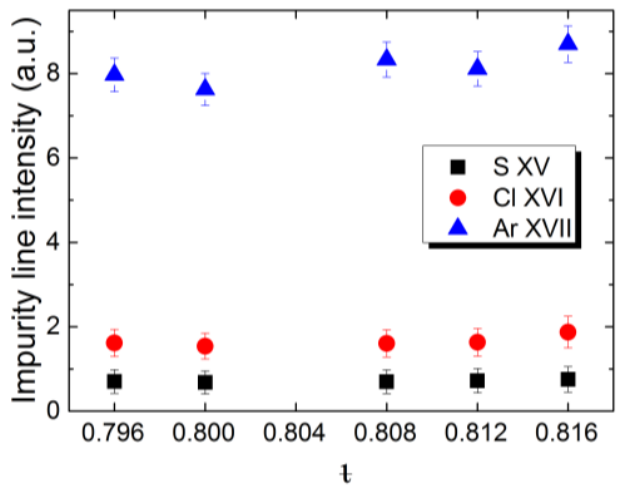

Fig. 4 Intensity of S XV at $2.460 \mathrm{keV}, \mathrm{Cl} \mathrm{XVI}$ at $3.267 \mathrm{keV}$ and $\mathrm{Ar}$ XVII at $3.140 \mathrm{keV}$ lines from time integrated PHA spectra as a function of $\mathfrak{t}$ (iota on the magnetic axis), (PID 20160309.016, 018, 024, 026, 029).

No significant changes of the impurity intensities are observed in these iota scans. It should be noted that due to the limited energy resolution, the intensity of the PHA Ar lines also contains the dielectronic recombination/innershell excitation satellites as observed by the XICS spectrometer [22, 23], but this does not affect the overall trend. A clear difference in the impurity intensity, however, is observed when the level of the ECRH power is changed, which affects the bulk plasma properties. Fig. 5 shows Ar XVII line intensity as a function of iota on the magnetic axis for three different ECRH power levels. Fig. 6 presents the time evolution of the ECRH power and Ar XVII line intensity in the investigated discharges. An increase of Ar XVII line intensity is observed with increasing ECRH power. The highest line intensity is observed for $\mathrm{P}_{\mathrm{ECRH}} \sim 2 \mathrm{MW}$. A similar behaviour of the Ar signal is observed by the XICS diagnostic (see Fig.7). Switching the heating power down and up reveals a corresponding response of the Ar signal.

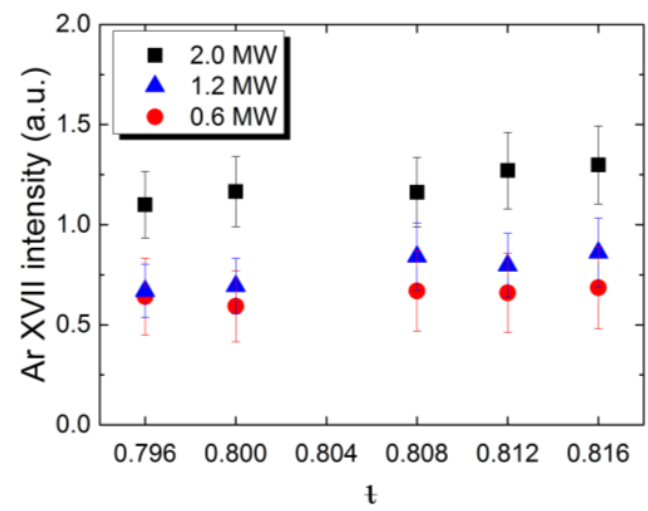

Fig. 5 Measured Ar XVII line intensity from PHA diagnostic as a function of $\mathfrak{z}$ (PID 20160309.016, 018, 024, 026, 029), for $\mathrm{P}_{\mathrm{ECRH}}=2 \mathrm{MW}, 1.2 \mathrm{MW}$ and $0.6 \mathrm{MW}$.



Fig. 6 Time evolution of measured Ar XVII at $3.140 \mathrm{keV}$ line intensity from PHA diagnostic for different iota on the magnetic axis (PID 20160309.016, 018, 024, 026, 029) with the ECRH power steps.

In order to obtain relative $\mathrm{Ar}, \mathrm{Cl}, \mathrm{S}$ impurity content in the plasma, numerical simulations have been performed. In these simulations, for a relative comparison of impurity contamination, coronal equilibrium was assumed and experimental profiles of $\mathrm{T}_{e}$ and $\mathrm{n}_{\mathrm{e}}$ from Thomson Scattering (TS) diagnostics [28] were taken. The use of corona equilibrium in the central part of the plasma is generally a good first approach for He-like ions which are distributed widely across the plasma cross-section due to the large step in its ionization energy to H-like states. Furthermore, the electron density profile is quite flat in the central part. The latter two aspects imply that small changes due to transport will not change the fractional abundances substantially. Fig. 
8 shows the response of relative concentrations of $\mathrm{Ar}$ (c_Ar), S (c_S) and $\mathrm{Cl}$ (c_Cl) to the changes of the ECRH power levels in a simulation made by the use of the upgraded version of RayX code [18]. The behaviour of $\mathrm{Ar}$ is similar to that presented in Fig.6. A higher relative concentration of $\mathrm{Ar}$ is observed in the plasma in comparison to $\mathrm{S}$ and $\mathrm{Cl}$.

As it can be seen in Fig. 9, showing experimental $T_{e}$ and $\mathrm{n}_{\mathrm{e}}$ profiles at $\mathrm{t}=0.3 \mathrm{~s}, 0.6 \mathrm{~s}$ and $0.9 \mathrm{~s}$, corresponding to the three ECRH power steps (as in PID 20160309.016, 018, 024, 026, 029 cf. Fig. 6), plasma profiles for different iota are highly reproducible. The fitting function $Y=$ $y_{o}\left(1-\left(r_{e f f} / a\right)^{n}\right)^{m}$ (were: $\mathrm{y}_{o}$ - central value of fitted parameter, $r_{\text {eff }}$ - effective radius, a - minor plasma radius, $\mathrm{n}$ and $\mathrm{m}$ - fitting parameters) to the corresponding experimental $\mathrm{T}_{\mathrm{e}}\left(\mathrm{r}_{\mathrm{eff}} / \mathrm{a}\right)$ and $\mathrm{n}_{\mathrm{e}}\left(\mathrm{r}_{\mathrm{eff}} / \mathrm{a}\right)$ profiles is used. In the performed experiment Core-Electron-RootConfinement (CERC) conditions [29], characterized by a centrally peaked $T_{e}$ profile, together with flat $n_{e}$ and $T_{i}$ profiles are observed. The electron temperature is higher than the ion temperature $\left(\mathrm{T}_{\mathrm{i}}(0) \sim 1.7 \mathrm{keV}\right)$, up to about 0.6 of the normalized minor radius. The CERC leads to decreased electron heat transport in the center, at low plasma densities resulting in $\mathrm{T}_{\mathrm{e}}>\mathrm{T}_{\mathrm{i}}$ [29]. The electronroot region extended radially with increasing $\mathrm{ECRH}$ power. As it can be seen in Fig. 1, central $T_{i}$ increases even when the heating power is decreased, but drops when the heating power is increased again. In CERC conditions the formation of a positive radial electric field $E_{r}>0$ in the plasma core is expected [29].

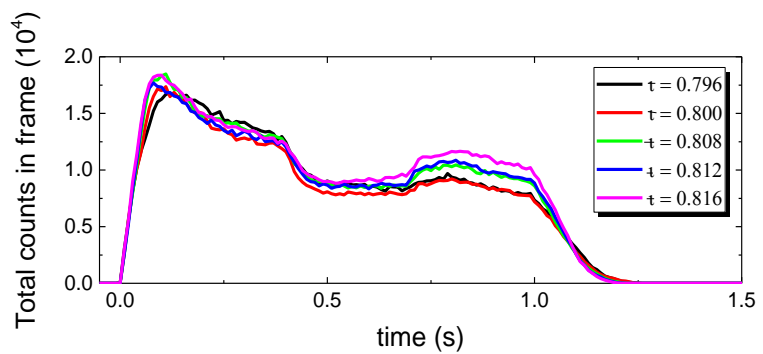

Fig. 7 The total Ar emission from the central line-integrated XICS channels for the shots with different iota (PID 20160309.016, 018, 024, 026, 029). Equivalent to the PHA measurement of Ar XVII line at $3.140 \mathrm{keV}$.

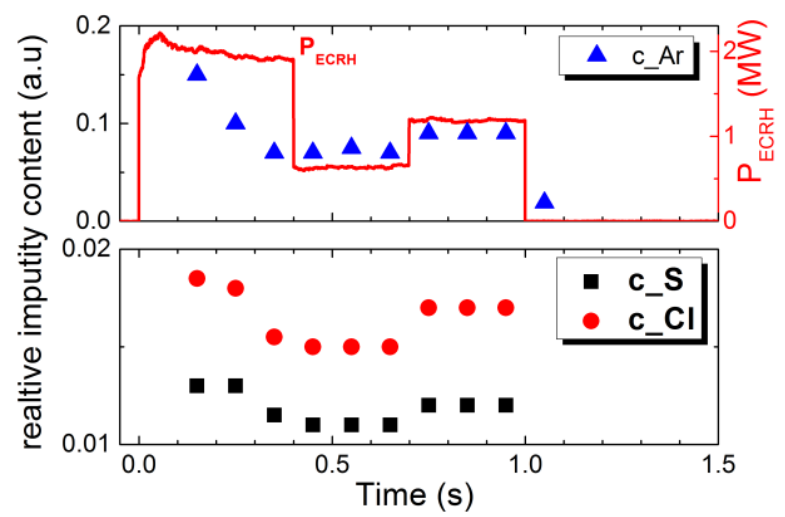

Fig.8 Estimated relative $\mathrm{Ar}, \mathrm{Cl}$, and $\mathrm{S}$ impurity content as a function of time with the ECRH power steps. (PID 20160309.024)

Both measurements [9, 22] and modeling [30] have shown that there is a positive radial electric field $E_{r}$ in the core. The $\mathrm{E}_{\mathrm{r}}$ profile has a strong influence on both the electron and ion fluxes. Large values of $E_{r}$ can reduce ion and electron neoclassical transport coefficients and enforce ambipolarity neoclassical particle fluxes. Under CERC conditions, $\mathrm{E}_{\mathrm{r}}$ is mainly given by the $T_{\mathrm{e}}$-gradient in the center of plasma because in this region $T_{e}>>T_{i}$ and the ion temperature profile is essentially flat $\left(\mathrm{T}_{\mathrm{i}} \nabla / \mathrm{T}_{\mathrm{i}} \approx 0\right)$; furthermore, the density profiles are flat in the center as well $\left(n \nabla / n<<T_{e} \nabla / T_{e}\right)$. Near the edge which is in the ion-root regime, $E_{r}$ generally plays a minor role. However, the lower temperatures and stronger density gradients can lead to a situation where the anomalous transport dominates over neoclassical transport

In order to examine the Ar content in the plasma core, Fig. 10 shows the line integrated Ar XVII intensity as a function of the mean $\mathrm{T}_{\mathrm{e}}$-gradient for OP1.1 magnetic configuration scans. It is seen that the change of the $\mathfrak{t}$ does not result within the error bar in clearly separated changes in the intensity of the Ar XVII line. However, there is a decrease of the Ar VXII line integrated intensity with $\left\langle\nabla \mathrm{T}_{\mathrm{e}}\right\rangle$. Since the plasma examined here reside in the CERC it is assumed that is also a dependence on $\mathrm{E}_{\mathrm{r}}$ indicated. Assuming the density of excited particle varies little during the heating periods $(\sim 9 \%)$, a stronger positive $\mathrm{E}_{\mathrm{r}}$ should result in larger outward impurity fluxes and, in turn, in a decrease of the Ar concentration. Yet, our observation indicate an increase in the intensity of Ar XVII line. This could be an indicator of a decreasing diffusivity with $E_{r}$ at low collisionality of the impurities and change in impurity transport. This way, the analysis allows making only a qualitative description of the observed phenomena. More accurate analysis, which takes into account actual impurity concentrations rather than the observed intensities, is required to validate this statement. In the future a collisional-radiative model including impurity transport is planned to be used for farther detailed analysis.

\section{Conclusions}

The performance of the W7-X stellarator during the first experimental campaign with limiter plasmas is explored using measurements from the PHA diagnostic, as well as Thomson scattering and data from the crystal imaging diagnostics XICS. Five scans of iota in the OP1.1 magnetic configuration were investigated. The discharge scenarios had highly reproducible $\mathrm{T}_{\mathrm{e}}$ and $\mathrm{n}_{\mathrm{e}}$ profiles. Analysis of PHA spectra shows that shifting the $5 / 6$ islands radially inward does not strongly impact the core impurity behavior. First experience with the magnetic island divertor will be gained in the next operational phase OP 1.2 at W7-X.

\section{Acknowledgments}

This work has been carried out within the framework of the EUROfusion Consortium and has received funding from the Euratom research and training programme 2014-2018 under grant agreement No 633053. The views and opinions expressed herein do not necessarily reflect those of the European Commission. This scientific work was partly supported by Polish Ministry of Science and Higher Education within the framework of the scientific financial resources in the years 2014-2018 allocated for the realization of the international co-financed project. 

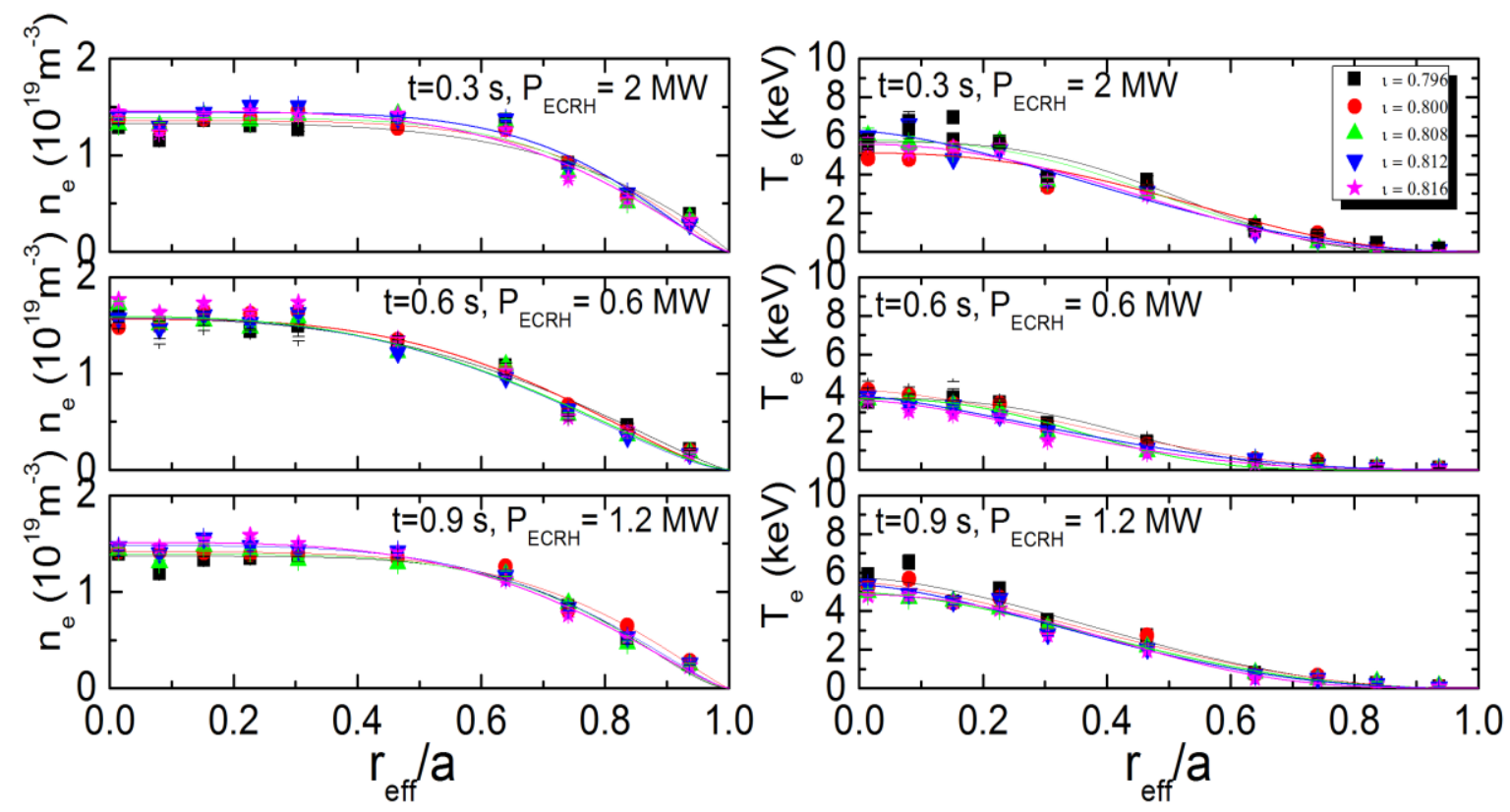

Fig. 9 Spatial distribution of density and temperature profiles taken from the Thomson Scattering diagnostic at 3 different steps of ECRH power, for OP1.1 discharges with different iota on the magnetic axis (PID 20160309.016, 018, 024, 026, 029).

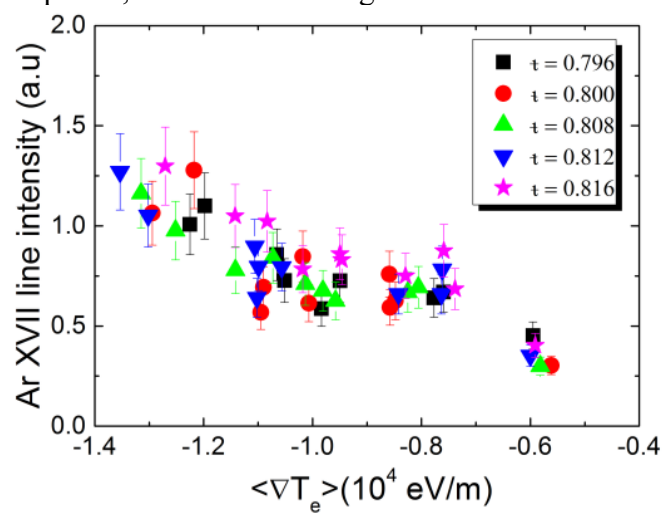

Fig. 10 The line integrated Ar XVII intensity as a function of the mean $\mathrm{T}_{\mathrm{e}}$-gradient for the scans of the OP1.1 magnetic configuration (PID 20160309.016, 018, 024, 026, 029).

\section{References}

[1] R. C. Wolf et al., First plasma operation of Wendelstein 7-X, Nuclear Fusion 58 (2017) 102020

[2] T. Klinger et al., Performance and properties of the first plasmas of Wendelstein 7-X, Plasma Phys. Control. Fusion 59 (2017) 014018

[3] M. Krychowiak et al., Overview of diagnostic performance and results for the first operation phase in Wendelstein 7-X, Rev. Sci. Instrum. 87, 11D304 (2016)

[4] R. Köning et al., The Set of Diagnostics for the First Operation Campaign of the Wendelstein 7-X Stellarator, 2015 JINST 10 P10002

[5] M. Hirsch et al., Confinement in Wendelstein 7-X Limiter Plasmas, Nucl. Fusion 57 (2017) 086010

[6] J. Nührenberg, and R. Zille, Stable stellarators with medium $\beta$ and aspect ratio, Phys. Lett A 114 (1986) 129

[7] G. Grieger et al., Physics optimization of stellarators Phys. Fluids B 4 (1992) 2081

[8] J.M. García-Regaña et. al., Electrostatic potential variation on the flux surface and its impact on impurity transport Nucl. Fusion 57 (2017) 056004

[9] F. Effenberg, et al., Numerical investigation of plasma edge transport and limiter heat fluxes in Wendelstein 7-X startup plasmas with EMC3-EIRENE, Nucl. Fusion 57
(2017) 036021

[10] L. Stephey et al., Control of neutral particle fueling and exhaust by optimization of the edge magnetic structure in Wendelstein 7-X and HSX, 58th Annual Meeting of the APS Division of Plasmy Physics San Jose, California USA, 31st October 2016

[11] A. Dinklage et al., Core Confinement in Wendelstein 7-X Limiter Plasmas 43thEPS2016, ECA Vol. 40A O2.107,

[12] J. Knauer et al., A New Dispersion Interferometer for the Stellarator Wendelstein 7-X, Proc. 43rd EPS Conf. (Leuven, Belgium, 4-8 July 2016) P4.017 (http://ocs.ciemat.es/EPS2016PAP/pdf/P4.017.pdf)

[13] M. Hirsch et al., ECE measurements in WENDELSTEIN 7-X plasmas, Proc. 43rd EPS Conf. (Leuven, Belgium, 4-8 July 2016) P4.007

[14] A. Langenberg et al., Temporal Evolution of Temperature and Argon Impurity Density Profiles Observed by X-ray Imaging Spectrometer Measurements at Wendelstein 7X, Proc. 43rd EPS Conf. (Leuven, Belgium, 4-8 July 2016) $\mathrm{P} 4.014$

[15] T. Sunn Pedersen et al., Plans for the first plasma operation of Wendelstein 7-X, Nucl. Fusion 55 (2015) 126001

[16] M. Kubkowska et al., Laboratory tests of the Pulse Height Analysis System for Wendelstein 7-X JINST 10 (2015) P10016

[17] R. Konig et al., Status of the diagnostics development for the first operation phase of the stellarator Wendelstein 7X, Review of Scientific Instruments 85 (2014) 11D818

[18] S. Jabłonski et al. ,Simulation of pha soft x-ray spectra expected from W7-X, JINST 10 (2015) P10021

[19] N. Krawczyk et al., Commissioning and first operation of the pulse-height analysis diagnostic on Wendelstein 7-X stellarator, Fus. Eng. Design 123 (2017) 1006

[20] M. Kubkowska et al., First Results from the Soft X-ray Pulse Height Analysis System at Wendelstein 7-X Stellarator, Fus. Eng. Design (2018). https://doi.org/10.1016/j.fusengdes.2017.12.024

[21] N. Krawczyk et al., Electron temperature estimation using the Pulse Height Analysis System at Wendelstein 7$\mathrm{X}$ Stellarator, 13th International Symposium on Fusion 
Nuclear Technology (ISFNT), Kyoto, Japan, 25th September 2017

[22] N. Pablant et al., Investigation of initial plasma parameters on the Wendelstein 7-X stellarator using the $\mathrm{x}$-ray imaging crystal spectrometer, IAEA conference 2016 EX/P5-6

[23] A. Langenberg et al., Argon impurity transport studies at Wendelstein 7-X using $\mathrm{x}$-ray imaging spectrometer measurements Nucl. Fusion 57 (2017) 086013

[24] B. Buttenschön et al., Spectroscopic impurity survey in the first operation phase of Wendelstein 7-X, EPS2016, P4.012,http://ocs.ciemat.es/EPS2016PAP/pdf/P4.012.pdf

[25] V. Winters et al., Overview of the plasma-surface interaction analysis in the limiter phase of W7-X, Physica Scripta, PFMC-16 Topical Issue (2017)

[26] JET Joint Undertaking Progress Report 1984

[27] E. Kallne et. al, Observation of $\mathrm{H}$ - and He-like x-ray line emission in high density tokamak plasmas, Physical Review Letters 49 (1982) 330-333

[28] E. Pasch et al., The Thomson scattering system at Wendelstein 7-X, Rev. Sci. Instrum. 87, 11E729 (2016)

[29] M. Yokoyama et al., Core electron-root confinement (CERC) in helical plasmas," Nucl. Fusion 47, 1213 (2007)

[30] M. Landreman et.al., Neoclassical calculations for W7-X OP1.1 parameters $58^{\text {th }}$ Annual Meeting of the APS Division of Plasmy Physics San Jose, USA, 31 Oct. 2016 